\title{
Second-Order Rotational Effects on Observations of $\beta$ Cephei Stars
}

\section{A. Engelbrecht (jr)}

\section{Department of Physics, University of South Africa, PO Box 392, Pretoria 0001, South Africa}

Practically all published models of $\beta$ Cephei ( $\beta$ Cep) stars assume a spherical profile, despite the fact that many actual $\beta$ Cep stars display high rotation velocities. As a result of the centrifugally altered profile of the surface of a rapidly rotating star, the surface area as well as the surface normal of an arbitrary element on the stellar surface will be modified. Numerical integration over the observed surface elements produces the observed luminosity and radial velocity of such a star. The effect of including the centrifugal distortion of a rotating model $\beta$ Cep star on its observed characteristics was studied for inclination angles $(i)$ of $0^{\circ}, 22^{\circ} .5,45^{\circ}, 67^{\circ} .5$ and $90^{\circ}$ and equatorial rotation speeds of $0,80,160,240,320$ and $400 \mathrm{~km} / \mathrm{s}$, for pulsation modes of order $l=0$ to 4 .

Previous studies of the ratios between radial velocity variations and magnitude variations by Balona \& Stobie (1979) indicated that these ratios are independent of $i$. Results of the present study for the radial mode and the sectoral quadrupole mode are shown below. In each case, the bottom panel shows the results obtained assuming no centrifugal distortion, while the top panel shows what is obtained when centrifugal distortion is included. The increasing dependence of these amplitude ratios on $i$ with increasing rotation speed is clear. Both graphs used an adiabatically-derived value of -17.5 for the ratio of proportional flux variation to proportional radius variation (commonly called the $f$-ratio), and the spread of the graphs was found to vary with $f$, although the trend remained similar.

The overall results obtained may be summarised as follows: Provided that accurate values of $f$ are used, the method of calculating amplitude ratios of light and velocity variations may be used to determine either equatorial rotation speed or angle of inclination of a star, provided that the pulsation mode of the star has been identified and the complementary parameter ( $i$ and rotation speed respectively) are known. This application becomes increasingly useful as the rotation speed increases, provided that proper account (second-order in $\Omega$ ) is taken of centrifugal distortion of stellar profiles.

Spectral line profiles were also modelled for the above mentioned range of parameters. The main conclusion was that rotation speeds determined from rotational broadening are significantly underestimated (by up to $25 \%$ ) if centrifugal distortion of the stellar profile is disregarded, at least for rotation speeds higher than $250 \mathrm{~km} / \mathrm{s}$. This agrees with a finding of Hardorp \& Strittmatter (1968).

E. C. Reynhardt is thanked for travel assistance. 

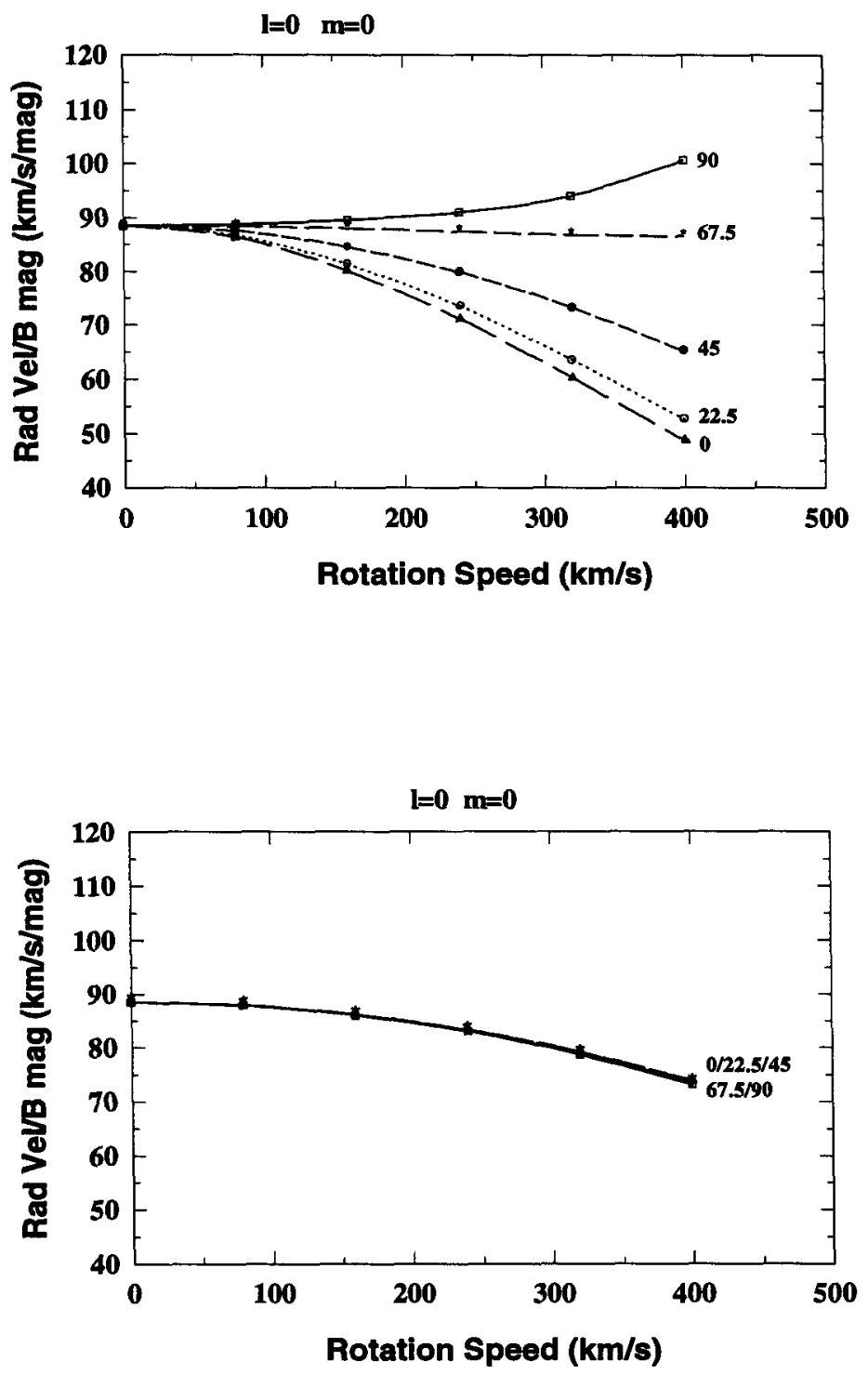

\section{References}

Balona, L.A., \& Stobie, R.S. 1979a, MNRAS, 187, 217

Balona, L.A., \& Stobie, R.S. 1979b, MNRAS, 189, 649

Hardorp, J., \& Strittmatter, P.A. 1968, ApJ, 153, 465 